\title{
Inflammatory Rhegmatogenous Retinal Detachment: Incidence and Outcome of Rhegmatogenous Retinal Detachments in Posterior Uveitis
}

\author{
Aliya Sultana \\ Department of Vitreoretina, Sarojini Devi Eye Hospital, Osmania Medical College, Hyderabad, Telangana State, India \\ Email address: \\ draliyasultana23@gmail.com

\section{To cite this article:} \\ Aliya Sultana. Inflammatory Rhegmatogenous Retinal Detachment: Incidence and Outcome of Rhegmatogenous Retinal Detachments in \\ Posterior Uveitis. International Journal of Ophthalmology \& Visual Science. Vol. 6, No. 2, 2021, pp. 89-93. \\ doi: $10.11648 /$ j.ijovs.20210602.15
}

Received: March 23, 2021; Accepted: April 14, 2021; Published: April 29, 2021

\begin{abstract}
BACKGROUND: Uveitis is one of the cause for blindness in our country in all age group of patients. Various causes of uveitis have been reported in our institute. All uveitis will not cause blindness, if appropriately managed. Blindness is mainly due to complications which occur as sequalae in these patients. Blindness can be due to anterior or posterior segment involvement. Early presentation and management will help in controlling the various complications. Rhegmatogenous retinal detachment is one of the cause in posterior uveitis patients which can occur due to changes in vitreous and retina. Uveitis can be infective or non-infective. AIM: Purpose of our study is to assess the incidence, management and outcome of rhegmatogenous retinal detachment in uveitis patients. MATERIALS AND METHODS: All patients of uveitis presented to our vitreo retina department were examined in detail, underwent various investigations to know the aetiology and managed based upon the clinical presentation. Retrospective study done by collecting patient data from old medical records. Duration of the study is 5 years, from June 2014 to June 2019. No of patients presented with posterior uveitis to our department during June 2014 to June 2019 were 610. All these patients underwent BCVA, Slit lamp examination, IOP, Fundus examination, b scan, OCT and documentation. Systemic examination done in all cases and also laboratory work up like RBS, CBP, MANTOUX TEST, HIV and serological testing if required, rheumatological work up in suspected cases. Patients with inflammatory rhegmatogenous retinal detachment underwent buckling plus pars plana vitrectomy and silicone oil endo tamponade. Fellow eye if required prophylactic laser done to the necrotic areas and when necrotic or tractional retinal breaks were present. RESULTS: Patients with mild PVR changes had good anatomical outcome, patients with severe PVR changes showed poor anatomical outcome. Visual outcome was poor in almost all cases. BCVA in all cases after oil removal was $\mathrm{CF} 1 / 2 \mathrm{mt}$ to $\mathrm{CF}$ $1 \mathrm{mt}$, some cases showed very poor outcome, BCVA in those cases was only perception of light in spite of good anatomical outcome. CONCLUSSION: Inflammatory retinal detachment is very serious condition in uveitis cases, PVR is definitely a poor prognostic factor, patient requires prolong endotamponade. Cases with prolong duration of uveitis history showed poor outcome. Even early presentation of cases also showed not much favourable visual outcome.
\end{abstract}

Keywords: Inflammatory Rhegmatogenous Retinal Detachment (IRRD), Uveitis, PVR, Ocular Toxoplasmosis, CMV Retinitis, Ocular Tuberculosis, Retinal Vasculitis, Retinal Breaks

\section{Introduction}

Uveitis can be infectious or non-infectious. Risk of retinal detachment is noted more in infectious uveitis cases. According to the published literature incidence of retinal detachment is $3 \%$. (Netherlands ophthalmologist Frank Kerkhoff and his colleagues found the incidence of rhegmatogenous retinal detachment in patients with uveitis to be approximately 3 percent [1]."

The rate of RRD in infectious uveitis appears to be more than three times greater than in non-infectious uveitis. PVR is one of the risk factor, incidence of PVR is high in infectious cases when compared to non-infectious cases, may be due to prolong activity of disease. 
Various pathogenic agents were noted in our study like toxoplasmosis, CMV retinitis, tuberculosis and toxocariasis. Syphilis is also one of the cause of the uveitis which can lead to retinal detachment. In our study syphilis patients were reported less to our department. Human herpes viruses, toxoplasmosis and syphilis are the organisms associated with RRD. The latter two organisms, however, are less likely to be associated with the widespread of retinal necrosis that follows infection by herpesclass viruses, and thus have a lower risk of RRD.

In patients with retinal detachment, location of breaks is not easy, because they are small breaks not visible properly, sometimes may be misdiagnosed as serous retinal detachment when there is absence of hydration or corrugation lines and the management can be delayed. If diagnosis is delayed, risk of PVR increases which is responsible for poor outcome.

PURPOSE: Aim is to study the incidence, management and outcome of rhegmatogenous retinal detachment in both infective and non-infective posterior uveitis cases.

\section{Materials and Methods}

All patients with uveitis were examined in detail, underwent both ocular and systemic examination.

5 years' study duration, retrospective study done from JUNE 2014 to JUNE 2019.

Patient data was collected from old medical records of our institute. The study was conducted with the approval of the institutional ethics committee with proper consent taken from the patients.

Inclusion criteria

Combined retinal detachment (tractional plus rhegmatogenous retinal detachment)

Rhegmatogenous retinal detachment

Both infective and non-infective cases

Exclusion criteria

Severe PVR

CMV retinitis cases with poor general condition

$46(7.5 \%)$ cases of 610 uveitis cases presented with RRD.

Number of cases underwent surgical procedure were 21 (45.6\%) out of 46 , remaining cases presented with severe PVR, pale disc, sclerosed vessels and some cases had no perception of light.

Infective aetiology noted in $19(90.47 \%)$ cases, $2(9.5 \%)$ cases were non infective.

Male gender showed preponderance compared to female patients.

$\begin{array}{ll}\text { Gender } & \text { No of cases } \\ \text { Male } & 17(80.95 \%) \\ \text { Female } & 4(19.04 \%)\end{array}$

Uveitis was reported in all age group of patients but retinal detachment was commonly seen between 25 to 35 age group patients.

BCVA in all these patients was hand motion / perception of light before surgery.

Based upon infective aetiology of various organisms detected by clinical manifestations and serological investigations.

$\begin{array}{ll}\text { Organism } & \text { No of cases } \\ \text { CMV Retinitis in Z + cases } & 9(47.36 \%) \\ \text { Ocular toxoplasmosis } & 4(21.05 \%) \\ \text { Ocular tuberculosis } & 3(14.28 \%) \\ \text { Intra ocular cysticercosis } & 2(9.52 \%) \\ \text { Herpes group of viruses } & 1(4.76 \%)\end{array}$

Clinical presentation of patients was full thickness necrosed retina with superficial retinal haemorrhages, moderate to severe vitritis, tractional bands, organised vitreous exudates, vitreous fibrosis, multiple healed pigmented scars with abnormal vitreo retinal adhesions, retinal necrosis extending from the periphery to the equator, pale retina with massive sub retinal exudation and mid peripheral necrosed lesions sparing extreme periphery.

Various investigations done to exclude the aetiology, like serological test, skin test, rheumatological work up, CBP, Chest X Ray and also high resolution CT scan chest.

After the investigations, patients with infective aetiology were treated with systemic steroids in immunocompetent patients and anti-microbial therapy (antibiotics/ anti-viral) drugs to control infection and inflammation, after two weeks of systemic steroids, surgical management was planned with consent of the patient. Prognosis was explained to patient and their attenders in their own language. Local steroid injections given in few $\mathrm{Z}+$ patients.

Circumferential band (240 band) used in most of the cases, buckling plus pars plana vitrectomy done with silicone oil endo tamponade, barrage laser done in all cases, drainage retinotomy done to drain sub retinal fluid in all cases, because visualisation of breaks were not possible due to tiny breaks.

\section{Results}

BCVA after silicone oil removal was CF $1 / 2$ to $1 \mathrm{mt}$ in all 16 cases $(76.19 \%)$, CFCF in $3(14.28 \%)$ cases and perception of light noted in two $(9.52 \%)$ cases.

Anatomical outcome was good in $16(76.19 \%)$ cases, 3 (14.28\%) cases showed persisting inferior retinal detachment due to severe inferior PVR, fibrosed thickened retina resisting flattening, iatrogenic breaks due to ischemic retina while releasing tractions and hyaloid stripping.

Inflammation and cell proliferation leads to the formation of tractional membranes on the epiretinal and sub retinal surfaces and also marked gliosis within the retina that leads to retinal shortening.

2 cases showed good outcome immediately after surgery, after 3 months' patient came with drop of vision, fundus showed re detachment of inferior retina involving posterior pole. These two patients required second procedure to flatten retina.

The visual outcomes among patients with RRD and uveitis were worse compared to outcomes in patients with nonuveitic RRD. Reason for this outcome is prolong inflammation. 
Proliferative vitreoretinopathy (PVR) is the main cause of retinal reattachment surgical failure in $5 \%-10 \%$ of rhegmatogenous retinal detachment (RRD) cases. PVR in uveitis cases will progress rapidly due to inflammation.

\section{Discussion}

Rhegmatogenous RDs featuring prominent inflammation which masquerade as a serous detachment is both diagnostic and therapeutic challenges [2]." Rhegmatogenous retinal detachment (RRD) seems to predominantly occur in patients with retinitis-associated posterior uveitis. Among cases of infectious uveitis, cytomegalovirus and varicella zoster virus were most commonly associated with RRD development. RRD in non-infectious uveitis was not found to be associated with any specific uveitis entity [3]." Risk of RRD is high (7.5\%) in our study compared to published literature, probably due to poor immune status, multi drug resistance, patient noncompliance and delayed presentation.

Infections cause about $20 \%$ of all uveitis cases, with the most common infectious aetiologies being herpetic infections and toxoplasmosis. Patients having infectious uveitis, CMV retinitis was predominantly associated with RRD development followed by ocular toxoplasmosis.

Vitritis causing vitreous degeneration, fibrotic changes leading to traction, vitreous shrinkage and posterior vitreous detachment may also contribute to RRD development.

Necrosis of the retina leads to retinal detachment in many patients. Retinal detachment occurs weeks to months after onset of the disease [4]." Full thickness, confluent areas of necrosis has risk of early onset of retinal detachment.

$5(23.80 \%)$ patients showed bilateral rhegmatogenous retinal detachment in infectious uveitis cases, all the 5 cases were $\mathrm{Z}+$ with CMV Retinitis involving all quadrants in one eye and moderate to severe CMV Retinitis with sparing of one quadrant of retina in other eye. Two cases of CMV Retinitis showed retinal detachment in one eye and peripheral full thickness necrosed retina in other eye. Remaining two patients showed retinal detachment in one eye and normal fundus in other eye.

CD4 count in all these patients was between 70 to 100 cells per cu mm. Patients with poor general condition were deferred for surgery. Due to vitritis location of breaks and extent of retinal detachment was not clearly visible. B Scan was done in all these patients to see the extent of retinal detachment.

One case of CMV retinitis was given intra vitreal ganciclovir injection, next day post injection patient developed rhegmatogenous retinal detachment. RRD may be due to vitreous incarcerated in the injection site caused traction on the thin ischemic retina leading to retinal break. When we plan intra vitreal injections in CMV retinitis cases better to inject at the site where retina is healthy.

Most common intra operative complication in these patients were iatrogenic breaks due to ischemic retina. Surgical outcome that is reattachment rate was good only in 4 cases (44.44\%), 2 cases $(22.22 \%)$ showed re detachment of retina after 3 months, these 2 cases developed star folds near the inferior arcade leading to retinal elevation, folds were released under silicone oil. Other 2 cases $(22.22 \%)$ had persisting inferior retinal detachment due to thickened retina, could not be flattened, vitreous base dissection was very difficult, hyaloid was tightly adherent due to inflammation, stripping of hyaloid caused full thickness large breaks at the periphery. Post-operative PVR changes in these cases occurs when the inflammation persists even after surgery for prolong period.

Visual outcome even after reattachment surgery is poor due to sclerosed retinal vessels, pale ischemic retina, may be the physiological function of retina is extinguished due to ischemic retina.

Cases of CMV retinitis should be followed frequently to prevent the risk of RRD. $30 \%$ of patients with CMV retinitis can develop RRD [5]." In our study maximum number of cases presented with inflammatory RRD were CMV retinitis related RRD.

All these patients should be followed regularly based on the CD4 count, if patients CD4 count is less than 50 cells/cu $\mathrm{mm}$ follow up is every 3 months, if less than $200 \mathrm{cells} / \mathrm{cu} \mathrm{mm}$ yearly twice, if more than 200 cells/cu $\mathrm{mm}$ at least yearly once [6]." Frequent examination in these cases can prevent blindness.

Second cause in our study was ocular toxoplasmosis, vitreous is firmly attached to the retina at the old healed retino choroiditis scar, vitreous retraction produces retinal holes and subsequent detachment of the retina. Clinically ocular toxoplasmosis presents as focal necrotising retinitis associated with old pigmented scars usually described as satellite lesion [7, 8]."

Tuberculosis (TB) has remained endemic in Asian countries with India, a large population size, accounting for nearly $20 \%$ of the global tuberculosis burden. Though ocular tuberculosis is one of the most common infective cause of uveitis in our study, retinal detachment in tuberculous uveitis cases reported only in four patients. Three patients presented with combined retinal detachment (tractional and rhegmatogenous retinal detachment) only one patient presented with rhegmatogenous retinal detachment, breaks were not visible, fellow eye showed signs of active retinal vasculitis. All these four patients were between 25 to 30 years old.

Incidence of disease in the fellow eye is very high. Due to poor surgical and visual prognosis in eyes with this type of detachment; the fellow eye requires special attention. We recommend periodic examinations at six month intervals and we instruct the patient to report immediately if any suspicious symptoms in the fellow eye occurs [9]."

Retinal detachment can also occur due to traction of epiretinal membranes which form due to inflammation leads to retinal breaks. Contraction of the epiretinal membrane causes a tangential traction on the retinal necrosis sites, the formation of retinal breaks and rhegmatogenous retinal detachment may occur [10]."

Release of inflammatory cytokines seen with rhegmatogenous $\mathrm{RD}$ and cytokines mediate the inflammatory 
features like vitreal membrane formation and retinal traction. In cases of rhegmatogenous RDs with significant inflammation, systemic corticosteroids should be considered to control perioperative inflammation for the favourable post-surgical outcomes. Heavy silicone oils use in inflammatory rhegmatogenous retinal detachment has good outcome is mentioned in literature because these patients require prolong tamponade, actually heavy silicone oil will cause intra ocular inflammation when compared to standard silicone oils. Mechanisms involved in the inflammatory response after the use of heavy tamponade is toxicity due to impurities or instability of the agent, direct toxicity and immunogenicity, oil emulsification, and mechanical injury due to gravity [11]."

Two cases of bilateral intra ocular cysticercosis presented to the retina department, both of them were young adults, pork eaters, had even neuro cysticercosis, fundus examination of one patient showed vitritis, multiple vitreous bands, sub hyaloid cyst with active larvae in right eye and left eye showed complicated retinal detachment. Before surgery patient treated with oral steroids for two weeks and albendazole for one week. Patient was explained regarding prognosis in left eye, deferred surgery in left eye and underwent vitrectomy and removal of sub hyaloid cyst in toto with vitrector in aspiration mode and turned to cutting mode carefully without dropping the cyst in the vitreous cavity, to prevent the release of toxins.

Other patient was also young male presented with both eyes complicated retinal detachment, surgery planned in one eye which had better prognosis compared to the other eye. Systemic steroids and albendazole started already by the neuro physician, vitrectomy done under guarded visual prognosis, silicone oil injected, breaks were not visible due to vitreous tractional bands, retina was fragile, intra operative multiple inferior retinal breaks occurred at the time of vitreous base dissection, first operative day there was minimal sub retinal fluid inferiorly, barrage laser was not possible due to elevated retina inferiorly, after two weeks patient presented with retinal folds near the inferior vascular arcade causing macula off. Second procedure planned to peel the epi retinal membrane, done and tried to flatten the retina. After two months' patient presented with migration of silicone oil in sub retinal space causing retinal detachment. Persisting inflammation after surgery will cause PVR changes which will lead to retinal redetachment [12]." Both anatomical and visual prognosis are poor in these patients.

Viral infections such as dengue and chikungunya are highly endemic in the Asia Pacific countries, in our study cases of dengue presented with vitreous haemorrhage, orbital haemorrhage and haemorrhagic retinal detachment, whereas chikungunya cases presented with focal retinitis involving mostly posterior pole following fever and arthritis, retinal detachment in these cases were not reported in our institute.

Some of the case reports showed RRD in VKH and sarcoid uveitis cases, we did not notice RRD in our institute. VKH was one of the most common aetiology of posterior uveitis seen in our study, risk of RRD in VKH was almost nil in our study. All these patients showed serous retinal detachment which was resolved with systemic steroids and immunosuppressive therapy. Sarcoidosis is a multisystem inflammatory disease which effects multiple organs, difficult to differentiate from ocular tuberculosis which is endemic in India due to clinical similarities [13]." Differentiating these two conditions is really a challenge, particularly in countries with high prevalence of TB. Combination of clinical, radiologic, and laboratory tests are used to make a diagnosis of sarcoidosis [8]. Only one female case of sarcoid choroidal granulomas in both eyes reported in our institute, which we diagnosed as tuberculous choroidal granulomas and referred to physician to exclude miliary tuberculosis and start ATT. Patient used steroids and ATT for three months, there was no response, the choroidal granulomas were same in size and number, sarcoid was suspected and referred to rheumatologist for further management. Possibility of misdiagnosis is high due to masquerading tuberculosis.

Success of surgery always depends on the PVR changes in cases of retinal detachment, PVR is a complex process causes ischemic tissue damage, but also inflammation and proliferation of several types of local cells and production of local factors [14]." In inflammatory RRD chronic retinal ischemia occurs due to breakdown of uveo vascular barrier leads to retinal atrophy.

\section{Role of Prophylactic Barrage Laser in Fellow Eye}

1) To control the progression of the retinitis

2) To prevent the risk of RRD

Retinitis is seen activated even from old scars, so barrage laser at the edge of healed dry scar will not stop from reactivation of disease because activity depends on the viral load and immune status.

Role of barrage laser before the development of RRD can prevent for some period, not throughout life span. In our study one young adult male presented with CMV retinitis involving inferior quadrant, treated with intra vitreal ganciclovir, till the activity is ceased, and barrage laser done to the healthy retina $1 \mathrm{~mm}$ from the healed lesion. We followed for one year, there was no reactivation, he maintained 6/18 vision, after one year he lost follow up.

One middle age $\mathrm{Z}+$ female who was myopic showed RRD in one eye and retinal necrosis in fellow eye. Retinitis was seen almost 360 degrees in the peripheral retina, patient underwent 360 degrees' prophylactic barrage laser, double frequency $\mathrm{Nd}$ Yag laser done, 1-disc diameter from the lesion to stop the progression.

CMV related RRDs are very difficult to understand, in our institute both children and adults presented with exudative retinal detachment in the first visit, with in few days they reported back with RRD.

Barrage laser may help only to prevent the RRD for some time so that you can treat primary disease with intra vitreal ganciclovir, also in patients where macula detachment is not there. In patients with CMV related RRD vitrectomy and 
silicone oil endo tamponade helps to flatten the retina but the primary disease that is CMV retinitis cannot be treated properly due to the presence of oil in vitreous cavity. Role of barrage laser whether useful or not requires to do more number of cases and also need to follow for longer period.

\section{Role of Anti PVR Therapies}

Several drugs have been proposed as an adjunctive treatment for preventing postoperative.

PVR. These include 5-Fluorouracil (5-FU) LowMolecular-Weight Heparin (LMWH), Daunorubicin, 13-CisRetinoic Acid, Cyclin-Dependent Kinases, and Corticosteroids [15]."

Pre and post-operative PVR due to inflammation is one of the important cause for poor visual prognosis in these cases, to prevent the risk of post-operative inflammation we tried steroids through various routes like sub conjunctival and posterior sub tenon during pre-operative period and intra operative intra vitreal steroids. Few patients were advised oral steroids with tapered doses in pre and post-operative period.

We divided in to two groups A and B, group A no of patient was 12 , treated with steroids, remaining 9 patients who were included in group B were not given steroids before surgery. Visual outcome in both the groups was same after the use of intra operative steroids.

Many other drugs were mentioned in literature, as anti PVR therapies, but we used only corticosteroids in inflammatory rhegmatogenous retinal detachment. I think we should try other drugs to reduce the proliferative activity in the retina.

Control of intra ocular inflammation may not affect the outcome whether the eye is completely quiescent or not before surgery.

Sometimes chronic RRD masquerading severe exudative pan uveitis will make diagnosis difficult. One elderly female patient presented with severe anterior chamber reaction, media was hazy due to vitritis, retinal detachment details were not clear, misdiagnosed as exudative retinal detachment due to bullous elevation, breaks not seen due to vitritis, when other eye fundus examined, inferior lattice degeneration with Horse shoe tear near the edge of lattice noted. Axial length of both eyes were $27.5 \mathrm{~mm}$. Barrage laser done to the horse shoe tear and planned other eye surgery under guarded visual prognosis after basic laboratory investigations.

\section{Conclusion}

Number of cases what we operated are less, probably the reasons are many cases presented in late stage with poor general condition, few cases were misdiagnosed as serous detachment, some patients deferred management due to poor prognosis. Inflammation and infection of eye has poor prognosis especially when all quadrants of the retina are affected compared to one or two quadrants of affected retina. Control of inflammation and infection is possible only when they present in the early stage. Management in cases of delayed presentation had poor anatomical and visual outcome.

\section{References}

[1] Rhegmatogenous retinal detachment and uveitis. Kerkhoff FT, Lamberts QJ, van den Biesen PR, Rothova A. Ophthalmology 2003; 110: 2: 427-31.

[2] Rhegmatogenous retinal detachment masquerading as exudative pan uveitis with intense anterior chamber inflammatory reaction. Ashlin S. Joye, Robert B. Bhisitkul, Daniel D. S. Pereira, John A. Gonzales. American Journal of Ophthalmology Case reports 2020; 18: 100618.

[3] Rhegmatogenous retinal detachment in uveitis. De Hoog et al. Journal of Ophthalmic Inflammation and Infection 2017; 7: 22

[4] Uveitis: Pathogenesis, Clinical presentations and Treatment Murtaza Mustafa, P. Muthusamy, SS. Hussain, SC. Shimmi, MM. Sein. IOSR Journal of Pharmacy 2014; 4; 42-47.

[5] John X Wong, Elizabeth P Wong and Stephen C Teoh. Outcomes of cytomegalovirus retinitis-related retinal detachment surgery in acquiredimmunodeficiency syndrome patients in an Asian population. BMC Ophthalmology 2014; 14: 150.

[6] E. L. Chuang and j. L. D avis. Management of retinal detachment. Associated with cmv retinitis in aids patients. Eye 1992; 6; 28-34.

[7] F. Kianersi, Naderi beni, H. Ghanbari, F. Fazel. Ocular toxoplasmosis and retinal detachment: five case reports. European Review for Medical and Pharmacological Sciences 2012; 16: 84-89.

[8] Francisco Virmond Moreira, Andressa Moreira Iwanusk, Augusto Radünz do Amaral, Mário Junqueira Nóbrega. Surgical outcomes of rhegmatogenous retinal detachment associated with ocular toxoplasmosis. Arq Bras Oftalmol. 2018; 81: 281-285.

[9] Rhematogenous retinal detachment complicated by severe intraocular inflammation, hypotony, and choroidal detachment. William h. Jarrett, ii, mdtr. Am. Ophth. Soc. Vol. Lxxix, 1981.

[10] A Case of Rhegmatogenous Retinal Detachment at Late Stage following Endogenous Bacterial Endophthalmitis. Daisaku Kimuraa, Takaki Satoa Hiroyuki Suzukia, Case Rep Ophthalmol 2017; 8: 334-340.

[11] Francesco Morescalchi, Ciro Costagliola, Sarah Duse et al. Heavy Silicone Oil and Intraocular Inflammation. BioMed Research International Volume 2014, Article ID 574825, 16 pages http://dx.doi.org/10.1155/2014/574825.

[12] IS Jain, SP Dhir, PR Chattopadhaya, Pravin Kumar. Ocular cysticercosis in North India. 1979; 27: 54-58.

[13] Sarcoidosis in tuberculosis-endemic regions: India. Kalpana Babu Journal of Ophthalmic Inflammation and Infection 2013; 3: 53 .

[14] Pathophysiology of Proliferative Vitreoretinopathy in Retinal Detachment. Justus G. arweg, Christoph Tappeiner Surv Ophthalmol 2013; 58: 230.

[15] Dexamethasone implant in silicone oil: In vitro behaviour. Flores Villalobos et al. Int J Retin Vitr 2018; 4: 24. 